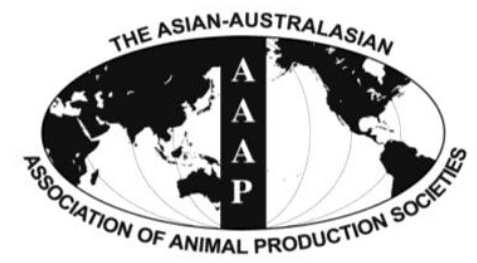

Asian-Aust. J. Anim. Sci.

Vol. 25, No. 6 : 886 - 894

June 2012

www.ajas.info

http://dx.doi.org/10.5713/ajas.2011.11380

\title{
Effects of Outdoor Housing of Piglets on Behavior, Stress Reaction and Meat Characteristics
}

\author{
Tomohiro Yonezawa, Asahi Takahashi ${ }^{1}$, Satomi Imai ${ }^{2}$, Aya Okitsu $^{2}$, Sonomi Komiyama ${ }^{1}$, \\ Mami Irimajiri ${ }^{2}, *$, Akihiro Matsuura ${ }^{2}$, Atusi Yamazaki ${ }^{1}$ and Koich Hodate ${ }^{1}$ \\ Laboratory of Veterinary Physiology, School of Veterinary Medicine, Kitasato University, Aomori 034-8628, Japan
}

\begin{abstract}
Well-designed housing systems are important from the viewpoint of animal welfare and improvement of meat production. In this study, we investigated the effects of outdoor housing of pigs on their behavior, cortisol levels, and meat characteristics. Two groups that were born and raised in a spacious outdoor pen $(4 \times 10 \mathrm{~m}$ for every two sows $)$ or a minimum-sized standard pen in a piggery $(1.9 \times 2.2 \mathrm{~m}$ for every sow $)$ were studied. When their behaviors at the age of 2 to 3 wk were observed, the number of rooting episodes tended to be larger $(p=0.0509)$ and the total time of rooting tended to be longer $(p=0.0640)$ in the outdoorhoused piglets although the difference was not significant. Basal salivary cortisol levels of the outdoor piglets at the age of 4 wk were significantly lower than those of the indoor piglets $(5.0 \pm 0.59 \mathrm{ng} / \mathrm{ml} \mathrm{vs.} 11.6 \pm 0.91 \mathrm{ng} / \mathrm{ml}, 30 \mathrm{~min}$ after treatment), although their plasma cortisol levels were similar $(53.3 \pm 3.54 \mathrm{ng} / \mathrm{ml}$ vs. $59.9 \pm 4.84 \mathrm{ng} / \mathrm{ml}, 30 \mathrm{~min}$ after treatment). When the ears were pierced at weaning, plasma and salivary cortisol levels were increased in both groups, even at $15 \mathrm{~min}$ after piercing. However, the increase in the outdoorhoused group was significantly less than that in the indoor-housed group. Throughout their lives, body weight and daily gain of the pigs were not significantly different between the two groups. In a meat taste preference test taken by 20 panelists, saltiness, flavor, and color of the outdoor-housed pork were found to be more acceptable. Moreover, when an electronic taste-sensing device was utilized, the C00 and CPA-C00 outputs $(3.78 \pm 0.07$ and $-0.20 \pm 0.023)$, which correspond to compounds of bitterness and smells, respectively, were significantly lower in the outdoor-housed pork (5.03 \pm 0.16 and $-0.13 \pm 0.009)$. Our results demonstrate that the outdoor housing system for piglets induces natural behaviors such as rooting and suppresses the strongest stress reaction of piglets, which could be important for animal welfare. Moreover, the outdoor housing system might change muscle characteristics and improve pork bitterness, flavor, and color. These changes may be preferred by consumers, increasing the sale of these meats. (Key Words: Environmental Enrichment, Salivary Cortisol, Taste Preference, Electronic Taste-sensing System, Animal Welfare)
\end{abstract}

\section{INTRODUCTION}

Well-designed housing systems for livestock minimize their stress levels and are important from the viewpoint of animal welfare. In intensive husbandry, pigs are exposed to various stressors (Jensen, 1994). A barren environment can impair the ability of pigs to cope with social and nonsocial challenges (O'Connell et al., 2004). In contrast, pigs housed in enriched pens have been shown to vocalize less, have a

\footnotetext{
* Corresponding Author: Mami Irimajiri. Tel: +81-176-23-4371 (ext. 377), Fax: +81-176-23-8703, E-mail: irimajim@vmas.kitasato-u.ac.jp

${ }^{1}$ Laboratory of Animal Feeding and Management, School of Veterinary Medicine, Kitasato University, Aomori 034-8628, Japan.

${ }^{2}$ Laboratory of Animal Behavior, School of Veterinary Medicine, Kitasato University, Aomori 034-8628, Japan.

Submitted Oct. 21, 2011; Accepted Dec. 25, 2011; Revised Jan. 8, 2012
}

longer latency period until movement, perform less overall locomotion during a 3-min human approach, and show a lesser degree of increase in salivary cortisol concentration after transport (Chaloupková et al., 2007). These findings suggest that environmental enrichment might reduce their stress reaction. However, the effects on piglets' behaviors and basal cortisol levels need to be investigated, and more precise time-series data on the effects of stress stimuli are required.

Not only animal well-being but also their growth efficiency and meat production might be affected by housing systems. Studies on housing systems for pigs have revealed effects on their performance and meat quality. The meat quality of pigs reared in enriched conditions was improved compared with that of pigs reared in barren conditions (Klont et al., 2001; Gentry et al., 2004). In those studies, although there were no differences in sensory tastes 
of the pork loin, various alterations in the percentages of muscle fiber types were observed. These findings indicated that meat production could be altered by outdoor and enriched housing, but how consumers react to these tastes remains to be determined.

In this study, we investigated the effects of outdoor housing of pigs on their behaviors, stress reactions, and meat characteristics. We prepared two groups that were born and raised in a spacious outdoor pen or in a minimumsized standard pen. To determine the effects of housing on their well-being, behavioral activities and salivary cortisol levels of the piglets were examined. A taste preference test was performed with the use of an electronic taste-sensing device to determine meat quality (Toko, 1996; 1998).

\section{MATERIALS AND METHODS}

\section{Animal handling and processing}

All procedures, including animal housing and care, were conducted according to the Guidelines for the Care and Use of Laboratory Animals, School of Veterinary Medicine, Kitasato University, Japan. Four Berkshire and Duroc sows and 20 piglets of the sows were used in this experiment. All pigs in the study were clinically healthy without porcine reproductive or respiratory syndrome histories.

The study was conducted at Kitasato University Field Science Center Towada Farm. The pregnant sows were placed in a farrowing pen or the outdoor farrowing facility at least 7-d prior to the expected due date to habituate them to the new settings. One Berkshire sow farrowed eight piglets on September 14, 2010, and one Duroc sow farrowed nine piglets on November 10, 2010. These piglets were born and housed in the standard breeding piggery with a gestation pen on concrete slatted flooring $(1.9 \times 2.2 \mathrm{~m}$ per sow) until weaning. The piglets were categorized as the indoor-housed group. One Berkshire sow farrowed 10 piglets on June 13, 2010, and one Duroc sow farrowed eight piglets on June 9, 2010. These piglets were born and housed together in an iron-fenced open space $(4 \times 10 \mathrm{~m}$ for every two sows) built outdoors in a soiled area, about half of which was covered with a polyvinyl chloride pipe greenhouse roof, until weaning. The piglets were categorized as the outdoor-housed group.

Sows were allowed free access to food and water at both facilities. The piglets were allowed to suckle at any time. All pens were equipped with two feeders and two nipple waterers for each sow and her litter. Animals had free access to a specific feed at each stage (Buri-meal Maximum, Tohoku Shiryo, Japan, for sows, and Super Coro-meal Uruoi Gold X, Kashima Shiryo, Japan, for weaning piglets). Indoor pigs were placed in a wooden piggery with windows and mean temperature of approximately $20^{\circ} \mathrm{C}$ (range, $10.5^{\circ} \mathrm{C}$ to $27.8^{\circ} \mathrm{C}$ ) during the trial period. The air temperature for the outdoor pigs during the trial ranged from $13.6^{\circ} \mathrm{C}$ to $28.0^{\circ} \mathrm{C}$. A bedded area with a heat lamp was provided for the piglets so that they could go to sleep whenever necessary.

\section{Behavioral analyses}

Piglets' behaviors were video-recorded using Handy Cam HDR-XR550V (normal mode, Sony, Tokyo, Japan) for $1.5 \mathrm{~h}$ with the focal animal sampling method (Altman, 1974) at postpartum 2 to $3 \mathrm{wk}$. Eight piglets from the indoor group (four from the Duroc litter and four from the Berkshire litter) and 10 piglets from the outdoor group (five from the Duroc litter and five from the Berkshire litter) were selected. The selection was based on the weight of the piglets: mid-weight piglets were chosen. The measurement parameters were registered and analyzed on the basis of a report by Petersen, 1994. The following social behaviors were analyzed: lying motionless or staying stationary (resting), touching the feeder for food (feeding), rapid thrusts to the floor with the head or snout (rooting), and active behavior toward another pig (social interaction). The number of episodes of these behaviors and total time spent for each behavior were measured.

\section{Collection and cortisol assay of plasma and salivary samples}

At 4 wk of age, the ears of all piglets were pierced using self-piercing ear tags, as a stress stimulus. Piglets were gently hand-restrained by three investigators to draw blood from the jugular vein, and cotton buds were inserted into the mouth for saliva collection (pretreatment). An ear tag (Allflex medium laser tag; Allflex USA Inc., TX) was attached within $1 \mathrm{~min}$ of blood and saliva sampling. Saliva and plasma samples were collected 3 times, at 15, 30, and 60 min after the stress treatment, using the same method.

Blood samples were placed in tubes containing heparin, and plasma was separated after centrifugation $(400 \times g, 15$ $\min , 4^{\circ} \mathrm{C}$ ). Saliva was collected by allowing the piglets to chew on cotton buds for 5 min with a soft curb strap (Yonezawa et al., 2011). The investigators could pick them up without contamination of blood from oral mucosal abrasion or excessive stress by using the soft curb strap. Each cotton bud was centrifuged for $15 \mathrm{~min}$ at $400 \times \mathrm{g}$ to obtain an approximately $500-\mu 1$ saliva sample. The saliva and plasma samples were stored at $-30^{\circ} \mathrm{C}$ until cortisol assay.

Cortisol concentrations in the saliva and plasma were measured in duplicate with a commercial enzyme immunoassay kit (Cayman Chemical Co., Ann Arbor, MI, USA) according to the manufacturer's protocol. Saliva and plasma samples were diluted to 1:50 and 1:200, respectively, with the buffer for the assay. The average intra-assay and inter-assay coefficients of variation were $2.23 \%$ and $8.92 \%$, 
respectively. The sensitivities were 0.50 to $50 \mathrm{ng} / \mathrm{ml}$ for saliva and 2 to $200 \mathrm{ng} / \mathrm{ml}$ for plasma.

\section{Meat preference and tasting test}

Animal selection and processing: Piglets were weaned at 28 days, and two outdoor female Duroc piglets were raised in a $400 \mathrm{~m}^{2}$ open-space field with a straw bedding area as free-range-raised pigs. These pigs were processed at 6 months. Of the two Duroc pigs that were born and raised outdoors, one was selected for meat quality measurements. The selected pig weighed $124 \mathrm{~kg}$ on the day of processing. An indoor-housed Duroc pig (weight, $120 \mathrm{~kg}$ ) from the Kitasato Field Science Center Towada Farm that was born and raised at an indoor piggery was processed for comparison at 6 months. Both pigs were used for meat measurements and had eaten the same feed during all developmental stages.

Questionnaire and panelists: A survey by questionnaire on taste and meat preferences was carried out in one room at the Laboratory of Animal Behavior, Kitasato University, on January 13, 2011. Panelists were 20- to 30-year-old volunteer undergraduate and graduate students at Kitasato University $(n=20)$. They were trained 3 times before the actual test to evaluate 5 tastes and their preferences. Evaluation sheets were distributed to all participants, 19 (four males and 15 females) of who completed the questionnaire (95.0\%).

An original questionnaire was created with 9 questions for comparing preference for sliced meat prepared from the musculus semimembranosus of the indoor- and outdoorhoused pigs. The participants were blinded to which pork was outdoor-raised and which was conventionally raised. The 5-point Likert scale was used for taste testing (Gregson, 1962; McGough et al., 2006). The questions were categorized as follows: (A) taste scores for saltiness, sweetness, and umami taste of grilled pork when the panelists pinched their nostrils; (B) the same scores for boiled pork; (C) preference for flavor of the boiled pork without nostril pinching; and (D) preference for lean color when the pork was rare. To aid in the characterization of the survey population, the participants were asked about their age, sex, and interest in livestock animal welfare. In addition, the participants were asked their overall opinion about their choice of pork, from the viewpoint of a consumer.

Panel sample preparation: The meat was prepared from the musculus semimembranosus of the indoor- and outdoorhoused pigs. The pigs were transported to a commercial slaughterhouse (Towada Meat, Aomori, Japan) for processing. Carcasses were fabricated into wholesale cuts after 5 days of aging. All meat was deboned, cut into 1- to 2-mm slices, vacuum-packaged in oxygen-impermeable bags (CCP-BONABONA-BM-V05 and XP3008-00, Japan), and stored in a freezer $\left(-30^{\circ} \mathrm{C}\right)$ until they were used for sensory evaluation.

The panel samples were prepared in the test kitchen of the Laboratory of Animal Behavior, Kitasato University. Frozen slices were thawed in a refrigerator $\left(4^{\circ} \mathrm{C}\right)$ for approximately $24 \mathrm{~h}$. Approximately 20 slices were grilled on a hot plate at $200^{\circ} \mathrm{C}$ for $40 \mathrm{~s}$. Approximately the same amount was boiled in tap water at $100^{\circ} \mathrm{C}$ for $40 \mathrm{~s}$. After cooling for approximately $5 \mathrm{~min}$, these pieces were served to each participant.

\section{Taste-trait analysis}

The INSENT SA402B electronic taste-sensing system (INSENT, Tokyo, Japan) was used for taste-trait analysis. The system is composed of 10 taste sensors of polymer membranes that fix different lipids (Toko, 1996; 1998). Sensors CA0, CT0, C00, AE1, AAE, CPA-C00, CPA-AE1, and CPA-AAE are designed to respond to individual tastes of sourness, saltiness, bitterness, astringency, umami taste, aftertaste from bitterness, aftertaste from astringency, and richness from umami, respectively (Toko, 1996; 1998). The sample solutions were prepared from the musculus semimembranosus of the indoor- and outdoor-housed pigs according to the manufacturer's protocol. Briefly, the sample meat $(5 \mathrm{~g})$ was homogenized with distilled water $(45 \mathrm{ml})$ using a homogenizer. After filtration (No. 238h), the filtrate was diluted to 1:5 with distilled water. Each sample was measured in triplicate. All assays were performed once. The average intra-assay coefficients of variation for the sensors were $0.99 \%, 1.56 \%, 10.29 \%$, $20.55 \%, 1.19 \%, 17.61 \%, 12.54 \%$, and $34.31 \%$, respectively.

\section{Statistical analyses}

Data analysis and suitability of parametric analysis were done using SAS (SAS Inst. Inc., Cary, NC). Piglets' behavioral profiles and the preference results for their meat were analyzed using Mann-Whitney's $U$ test. Plasma and salivary cortisol levels were analyzed using 2-way repeatedmeasures ANOVA followed by Scheffe's post hoc test. The sensory outputs by SA402B were analyzed using Student's $t$ test. Statistical significance was assumed if the $p$ value was $<0.05$. The $\mathrm{p}$ value is shown in figures if it was less than 0.1 .

\section{RESULTS}

\section{Behavioral analyses}

No significant differences in weight or daily gain of the piglets were found between the two housing system groups throughout the piglets' lives. The average weights at the age of 4 wk were $7.52 \pm 1.65 \mathrm{~kg}(\mathrm{n}=8)$ for the indoor piglets and $7.83 \pm 1.00 \mathrm{~kg}(\mathrm{n}=10)$ for the outdoor piglets.

To determine the effect of housing system on behaviors, the piglets' behavioral parameters were observed $(n=8$ and 
10) for $1.5 \mathrm{~h}$ at the age of 2 to $3 \mathrm{wk}$ (Figure 1). Although there were no statistically significant differences between the two groups, the number of episodes and total time of rooting tended to be greater in outdoor-housed pigs than in indoor-housed pigs ( $p=0.0509$ and 0.0640 , respectively) but the difference was not significant. No differences in resting, feeding, or social interaction were found between outdoor- and indoor-housed pigs. Piglets rarely showed suckling behavior during the sampling period, and there were no significant differences between the two groups (data not shown).

\section{Plasma and salivary cortisol levels}

Plasma and salivary cortisol levels of the weaned piglets at the age of $4 \mathrm{wk}$ were determined to find differences in endocrinological stress reaction between the two groups (Figure 2). Time-series data for both plasma and salivary cortisol levels were obtained before and after ear piercing as a stress stimulus. Although pretreatment plasma cortisol concentrations were similar in the two groups, basal salivary cortisol levels of the outdoor piglets were significantly lower than those of the indoor piglets. After the stress treatment, plasma cortisol levels acutely increased in both groups. Salivary cortisol levels also increased in both groups $(53.3 \pm 3.54 \mathrm{ng} / \mathrm{ml}$ vs. $59.9 \pm 4.84 \mathrm{ng} / \mathrm{ml}, 30 \mathrm{~min}$ after treatment), but salivary cortisol increased to a significantly lower level in the outdoor-housed group $(5.0 \pm 0.59 \mathrm{ng} / \mathrm{ml}$ vs. $11.6 \pm 0.91 \mathrm{ng} / \mathrm{ml}, 30 \mathrm{~min}$ after treatment). These alterations were found $15 \mathrm{~min}$ after ear piercing and continued until $120 \mathrm{~min}$ after the stress. Salivary protein content was also measured. The protein contents of indoor- and outdoor-housed piglets were $2.42 \pm$ $0.38(\mathrm{n}=8)$ and $2.16 \pm 0.48 \mathrm{mg} / \mathrm{ml}(\mathrm{n}=10)$, respectively, which were similar and thus not significantly different $(\mathrm{p}=$ 0.67). Even when their cortisol levels were recalculated with individual protein content in the saliva, the adjusted salivary cortisol values in the outdoor group were significantly lower than those in the indoor group (data not shown).

\section{Meat preference and tasting test}

To evaluate the differences in taste characteristics of

A)
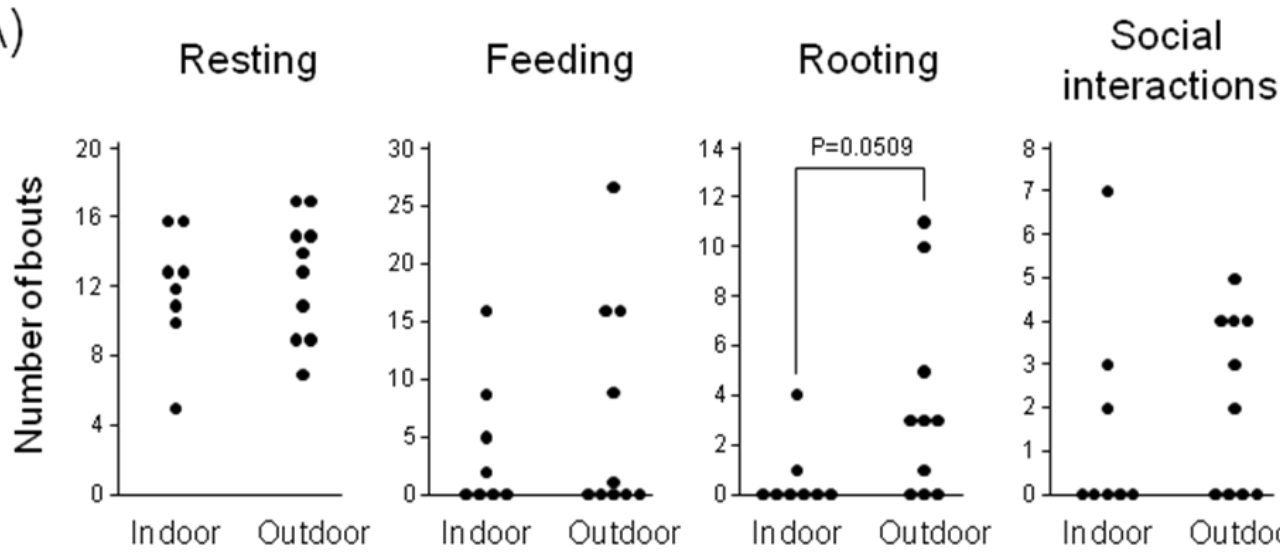

B)
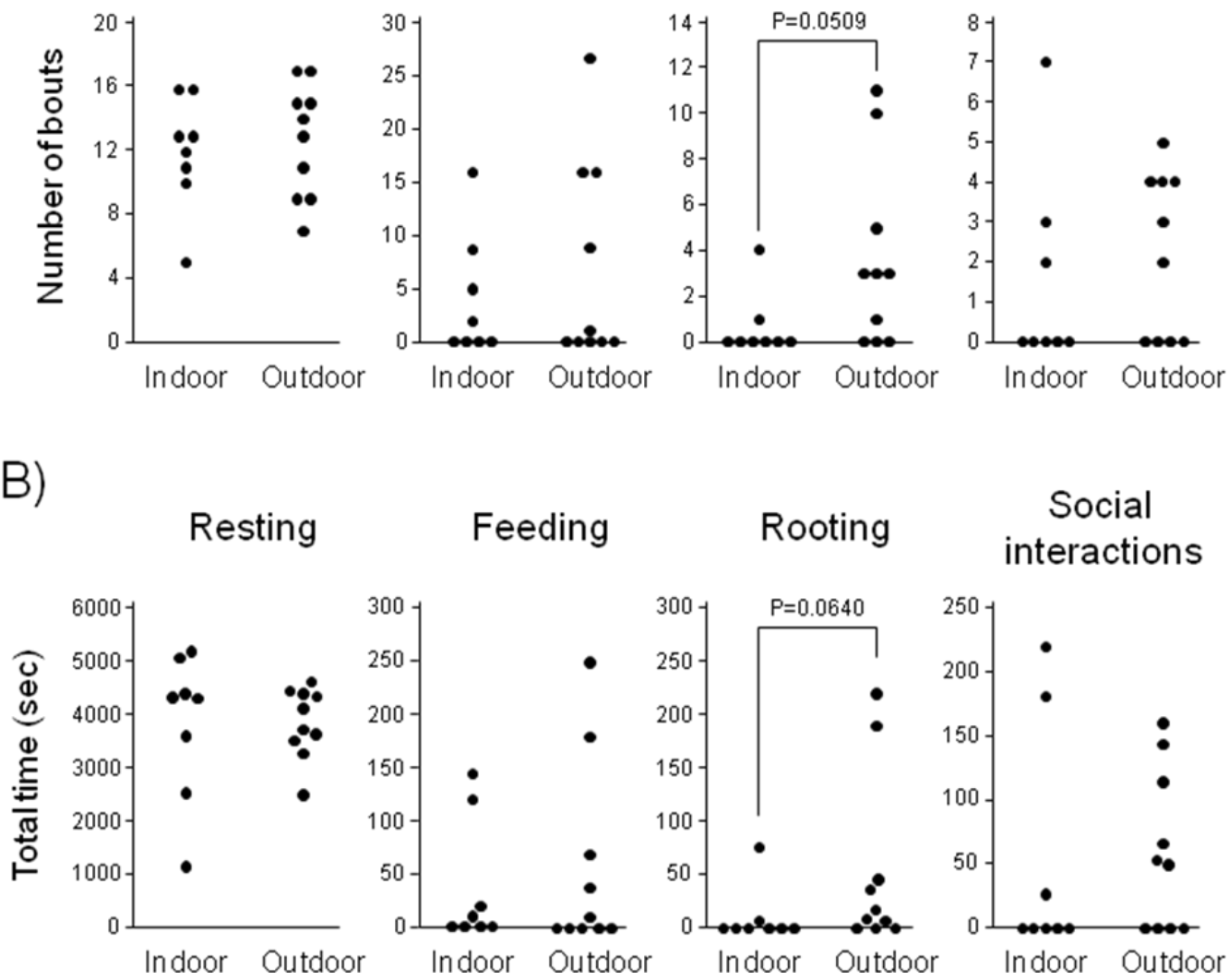

Figure 1. Scattered plots for behavioral profiles of piglets ( 2 to 3 wk old) housed in indoor or outdoor pens. These profiles were measured for $1.5 \mathrm{~h}$. Each plot indicates the individual score for the number of episodes (A) and total time spent for each behavior (B). Although there were no significant differences between the two groups $(p<0.05)$, the $p$ values are shown if they were $<0.1$. 

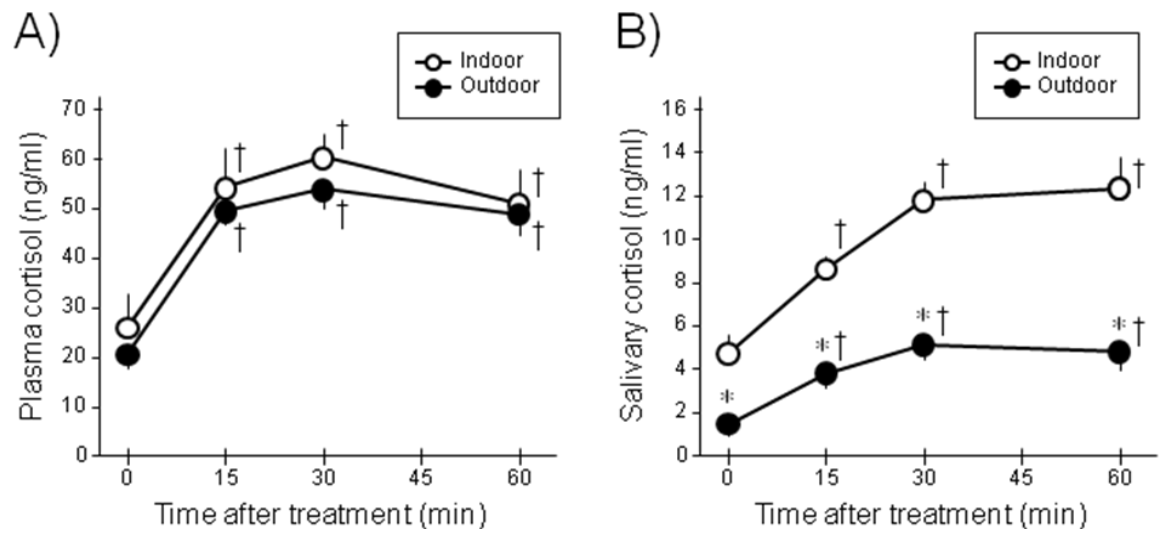

Figure 2. Plasma (A) and salivary (B) cortisol levels after stress exposure in indoor- or outdoor-housed piglets $(4 \mathrm{wk}$ old; $\mathrm{n}=8$ and $\mathrm{n}=$ 10, respectively). Time zero indicates the time at which the ears were pierced. The open and closed circles indicate the cortisol levels of indoor- and outdoor-housed piglets, respectively (means \pm SEM). Asterisks and daggers indicate statistical significance vs. indoor piglets and time zero, respectively $(\mathrm{p}<0.05)$.

pork meat between indoor- and outdoor-housed pigs, taste preference and a tasting test taken by 20 panelists using the electronic taste-sensing device were performed. The pigs used for these tests were processed at the age of 6 months. Their weight was $120-124 \mathrm{~kg}$, and their certificates of meat quality assessed by the slaughterhouse did not show any differences between the housing systems. The results from the 19 completed questionnaires were subjected to statistical analyses. Taste scores for the meat prepared from the musculus semimembranosus of the indoor- and outdoorhoused pigs are shown in Figure 3. Taste scores for saltiness, sweetness, umami taste, and tenderness of the grilled pork
A)

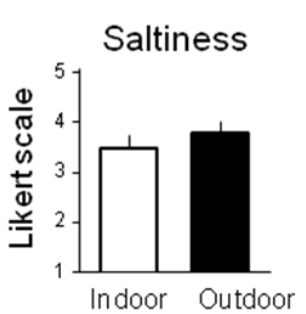

B)

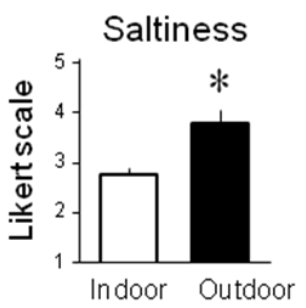

C)

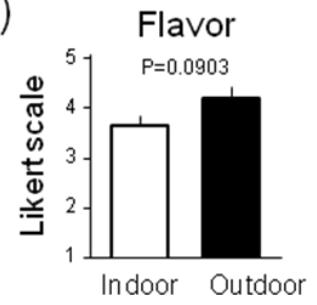

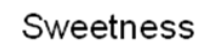
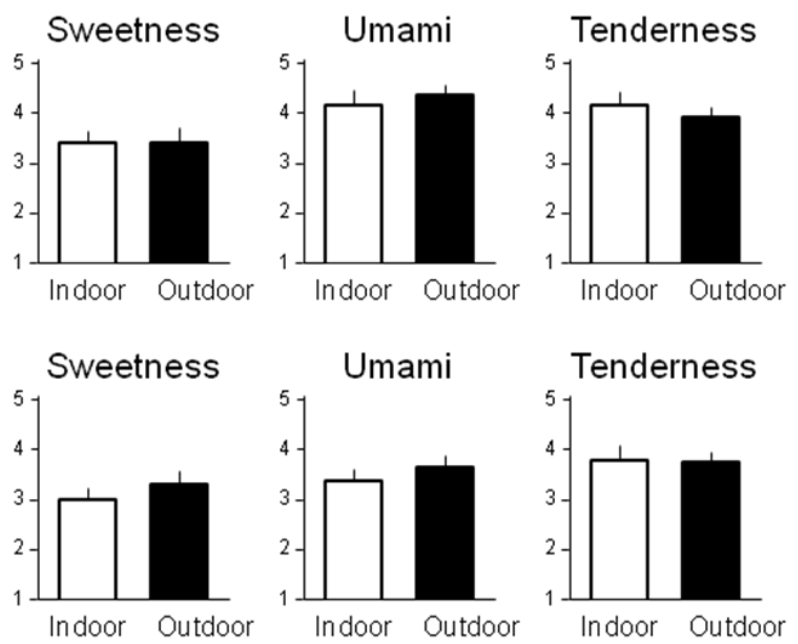

D)

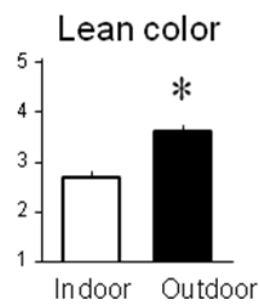

Figure 3. Taste scores for meat prepared from the musculus semimembranosus of the indoor- and outdoor-housed pigs $(\mathrm{n}=19)$. Columns and bars indicate mean and SEM, respectively. (A) Taste scores for saltiness, sweetness, umami taste, and tenderness of the grilled pork when the panelists pinched their nostrils. (B) Corresponding scores for the boiled pork. (C) Preference for flavor of the boiled pork without nostril pinching. (D) Preference for lean color when the pork was rare. Asterisks indicate statistical significance $(\mathrm{p}<0.05)$, and the $\mathrm{p}$ value is shown if it was less than 0.1 . 
(Figure 3A) and the same scores for the boiled pork (Figure 3B) were marked while the panelists pinched their nostrils. Panelists' preference for the flavor of the boiled pork without nostril pinching (Figure 3C) and lean color when the pork was rare (Figure 3D) was scored in the next section of the questionnaire. The saltiness score of the grilled pork and lean color score were significantly higher for the outdoor group than the indoor group. The flavor score of the boiled outdoor-housed pork was higher, but the difference was not significant $(p=0.0903)$. No significant differences were found for the other scores.

\section{Taste-trait analysis}

The electronic taste-sensing device INSENT SA402B, which is composed of several kinds of lipid/polymer membranes for transforming information on taste substances into electric signals (Toko, 1996; 1998), was utilized to evaluate the taste of the raw pork slices $(n=3)$. This system showed that the $\mathrm{C} 00$ and CPA-C00 outputs of outdoor-housed pork $(3.78 \pm 0.07$ and $-0.20 \pm 0.023)$ were significantly lower than those of indoor-housed pork (5.03 \pm 0.16 and $-0.13 \pm 0.009$, Figure 4). These outputs corresponded to direct bitterness taste and bitterness taste from aftertaste, respectively. The CA00, CT00, AAE, and CPA-AE1 outputs, corresponding to sourness, saltiness, umami, and aftertaste from astringency, respectively, showed no significant differences between the two groups. The AE1 and CPA-AAE outputs, corresponding to astringency and richness from umami, respectively, also showed no significant differences, although these outputs might be unreliable because the coefficient variances were $>20 \%$.

\section{DISCUSSION}

Previous studies on the effects of housing system on pig performance and meat quality have shown several differences in their phenotypes. Beattie et al. (2000) and Chaloupková et al. (2007) found that pigs reared in enriched environments had higher growth rates, heavier postmortem carcasses, and differences in meat $\mathrm{pH}$. In contrast, some studies have shown that free-range or enriched housing had no significant effects on carcass weight, meat percentage, or higher back fat thickness (Van der Wal et al., 1999; Klont et al., 2001). Although precise clarification of the reasons for the differences in results of those studies is required, one reported possibility is that climatic conditions could affect pig performance in an outdoor production system (Enfält et al., 1997; Sather et al., 1997). In the present study, body weight and daily gain of the pigs were not significantly different between the two groups. Nevertheless, the outdoor-housed piglets were slightly heavier than the indoor-housed piglets. This at least indicates that our outdoor housing conditions, which are characterized by high humidity and temperature but the presence of outdoor breeze, were accepted by or even beneficial for the piglets.

Since livestock housing systems are known to affect the behavior of livestock, these housing systems are also known to be important for animal welfare farming. In poorly housed conditions, the coping ability of pigs in the face of
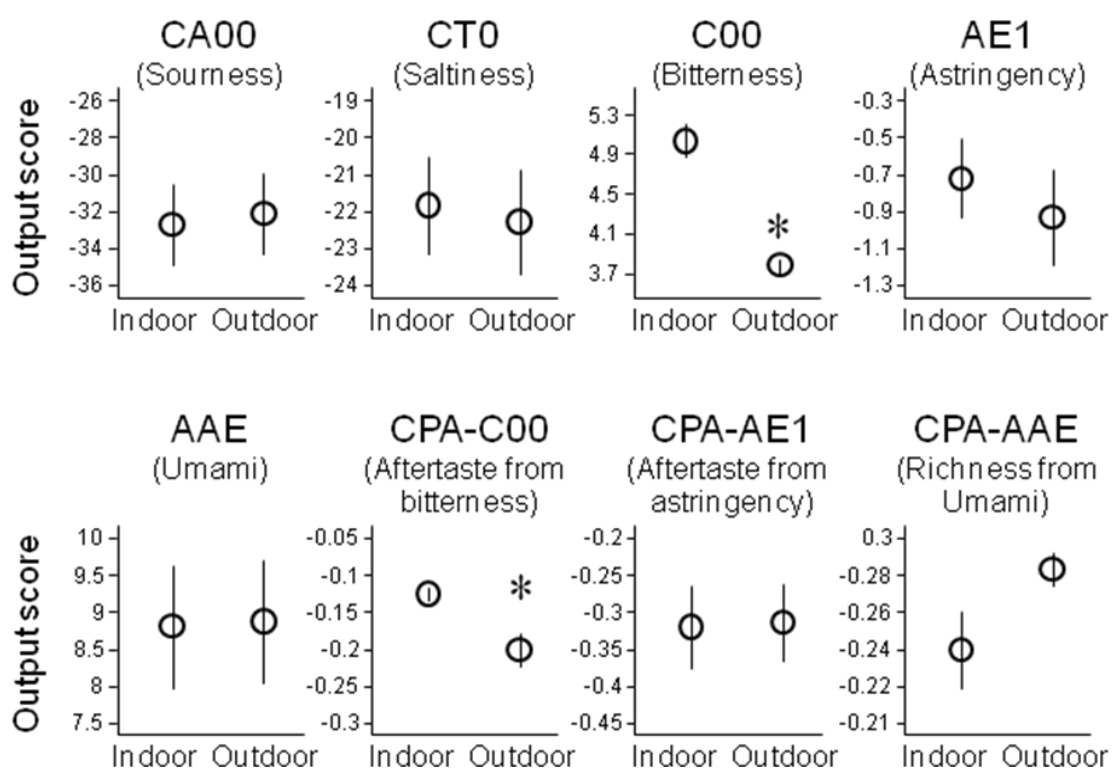

Figure 4. Taste-trait analysis of sample solutions prepared from the musculus semimembranosus of the indoor- and outdoor-housed pigs using an electronic sensing system (INSENT SA402B). Each output was designed to respond to the respective individual taste shown in parentheses. Each sensor output is shown in arbitrary units. Dots and bars indicate mean and SEM, respectively. Asterisks indicate statistical significance $(\mathrm{p}<0.05)$. 
social and nonsocial challenges has been shown to be impaired compared with that of pigs raised in enriched environments (Jensen, 1994; O'Connell et al., 2004). In this experiment, although behaviors associated with resting, feeding, or social interactions were not different between the housing systems, the number of episodes was significantly greater and the total time spent floor-rooting was significantly longer in outdoor-housed piglets than in indoor-housed piglets. Rooting the floor with the nose is compatible with a natural behavior exhibited by wild sows for excavating, mounding, and shaping the nest (Hartsock and Barczewski, 1997; Persson, 2008). These results suggest that outdoor-housed piglets could exhibit apparently natural behaviors such as rooting because the environmental limitation was partially removed. Allowing piglets to live in a free-range piggery might provide for their behavioral demands and needs. The World Organization for Animal Health announced that its member countries and territories mandated that the organization take the lead internationally on animal welfare as part of their 2001-2005 strategic plans (Matsuki, 2008; Sato, 2011). Our outdoor housing system may be one way of fulfilling this expectation.

Cortisol measurements have been used to evaluate stress reaction in animals as an indicator of welfare (Harbuz and Lightman, 1992; Yonezawa et al., 2009). In the present study, the basal salivary cortisol levels of outdoor-raised pigs were lower than those of indoor-raised pigs. After ear piercing, the increase in salivary cortisol in outdoor-housed pigs was also clearly smaller than that in indoor-housed pigs. This result corresponds to results of studies by De Jong et al. (2000) and Chaloupková et al. (2007) showing that salivary cortisol levels increased after stress stimuli in pigs reared in barren environments but did not increase to the same degree in pigs reared in enriched housings. We examined their salivary protein contents and found no difference between the two groups or change with time. This indicates that these cortisol alterations could not have been derived from changes in saliva production or release. Taken together, our results demonstrate that the outdoor housing system for preweaned piglets suppresses their strongest stress reaction, which could be important from an animal welfare viewpoint.

In this study, we measured not only salivary cortisol concentration but also plasma cortisol levels. Interestingly, although the salivary cortisol levels differed between the indoor and outdoor groups, the plasma cortisol concentrations were almost identical. As described above, salivary protein contents did not differ between the two groups or change with time, indicating that alterations in salivary cortisol levels were not affected by saliva production. This interesting discrepancy between plasma and salivary cortisol levels might reflect the tissue glucocorticoid-transporting mechanism. Cortisol is released into circulation from the adrenal glands; this release is induced by adrenocorticotropic hormone (ACTH), which is stimulated by corticotrophin-releasing factor. This series of interactions is called the hypothalamus-pituitary-adrenal (HPA) axis (Westphal, 1986). On the other hand, the transport of glucocorticoids from blood to target tissues mainly involves the balance of binding proteins, such as corticosteroid-binding globulin (CBG) (Klieber et al., 2007). Since plasma cortisol was not altered in this study, suppression of outdoor-piglets' stress reactions might be caused not by changes in the hormonal balance of the HPA axis but by expression of cortisol-binding proteins such as CBG. Further study is needed to identify any differences in CBG between the groups and the reason for the change in expression levels in pigs housed outdoors.

Since there is a rather negative relationship between stress and meat characteristics in pigs (Van der Wal et al., 1999), housing systems could also affect meat superiority. Indeed, the meat quality of pigs reared in enriched housings has been shown to be superior to that of pigs reared in barren conditions in terms of cooking loss, intramuscular fat content, and muscle characteristics (Klont et al., 2001; Gentry et al., 2002). In this study, to evaluate the effects of rearing conditions on meat quality, a taste preference test and mechanical measurement of sensory traits were performed.

In the taste preference test, almost all of the sensory scores were not significantly different between the two groups, somewhat corroborating the findings of Gentry et al. (2004). Nevertheless, the panelists stated that outdoor pig pork was saltier with more meat flavor. To identify more of these taste characteristics, we conducted an electronic tastesensing test. This test was established by Toko $(1996 ; 1998)$ to analyze nonvolatile compounds as taste components in foods by using polymer membranes. According to Chikuni et al. (2010), even the taste traits of different muscle types can be distinguished by this system. In this study, C00 and CPA-C00 outputs were significantly lower in the outdoorhoused pork. Since C00 and CPA-C00 outputs correspond to direct bitterness taste and bitterness taste from aftertaste, respectively (Toko, 1996; 1998), these data imply that outdoor housing should improve pork taste traits by reducing its bitterness. Unfortunately, we did not include any questions about bitterness in the questionnaire given during the taste preference test. The target candidates responsible for bitter taste in animal meats are iso- $\alpha$-acidlike compounds, which include some hydrophobic carbonyl compounds (Chikuni et al., 2010). Most of these compounds in meat are produced from hydrolytic degradation of lipids and processed by $\beta$-oxidation (Gandemer, 2002), which is thought to reduce the levels of these compounds in outdoor-housed pigs that have lower stress levels. Moreover, the carbonyl components of C00 
outputs are mainly responsible for the smell of meat (Macleod, 1994). This evidence corroborates the sensory preference data showing that the flavor score of outdoorhoused pork tends to be higher. All of these findings indicate that the outdoor pork could indeed have improved taste.

The panelists in this study also stated that the color of the meat of outdoor-housed pigs was redder. The effects of housing systems on muscle characteristics have been reported in some papers. The ratio of type I muscle fibers is reportedly augmented in outdoor-born pigs' muscles because of increased locomotion (Maltin et al., 1997; Gentry et al., 2002; 2004). Type I fibers are known to be redder than type II fibers; this could explain the difference in meat color observed in this study. These changes in pork tastes could have been derived at least partially from changes in muscle characteristics.

This study has demonstrated that rearing piglets in a spacious outdoor facility could be important for both the welfare of the pigs and for improving their meat quality. Although a clear explanation of the physiological mechanisms is needed, the outdoor housing system could be conducive for some natural behaviors such as rooting and suppress stress reactions. This system might also change muscle characteristics and improve pork flavor and color, increasing consumer preference for such meat.

\section{ACKNOWLEDGEMENTS}

Support for this project was provided by a Grant-in-Aid for Scientific Research from the Japan Society for the Promotion of Science. The authors are grateful to Mr. T. Nanbu and Dr. D. Watanabe at Kitasato University Field Science Center for management of animals.

\section{REFERENCES}

Altman, J. 1974. Observational study of behavior: sampling methods. Behaviour 49:227-267.

Beattie, V. E., N. E. O'Connell and B. W. Moss. 2000b. Influence of environmental enrichment on the behavior, performance and meat quality of domestic pigs. Livest. Prod. Sci. 65:71-79.

Chaloupková, H., G. Illmann, K. Neuhauserová, M. Tománek and L. Valis. 2007. Preweaning housing effects on behavior and physiological measures in pigs during the suckling and fattening periods. J. Anim. Sci. 85:1741-1749.

Chikuni, K., M. Oe, K. Sasaki, M. Shibata, I. Nakajima, K. Ojima and S. Muroya. 2010. Effects of muscle type on beef tastetraits assessed by an electric sensing system. Anim. Sci. J. 81:600-605.

De Jong, I. C., I. T. Prelle, J. A. Van De Burgwal, E. Lambooij, S. M. Korte, H. J. Blokhuis and J. M. Koolhaas. 2000a. Effects of environmental enrichment on behavioral responses to novelty, learning, and memory, and the circadian rhythm in cortisol in growing pigs. Physiol. Behav. 68:571-578.
Enfält, A. C., K. Lundstrom, I. Hansson, N. Lundeheim and P. E. Nystrom. 1997. Effects of outdoor rearing and sire breed (Duroc or Yorkshire) on carcass composition and sensory and technological meat quality. Meat Sci. 45:1-15.

Gandemer, G. 2002. Lipids in muscles and adipose tissues, changes during processing and sensory properties of meat products. Meat Sci. 62:309-321.

Gentry, J. G., J. J. McGlone, J. R. Blanton, Jr. and M. F. Miller. 2002. Impact of spontaneous exercise on performance, meat quality, and muscle fiber characteristics of growing/finishing pigs. J. Anim. Sci. 80:2833-2839.

Gentry, J. G., J. J. McGlone, M. F. Miller and J. R. Blanton, Jr. 2004. Environmental effects on pig performance, meat quality, and muscle characteristics. J. Anim. Sci. 82:209-217.

Gregson, R. A. M. 1962. A rating-scale method for determining absolute taste thresholds. J. Food Sci. 27:376-380.

Harbuz, M. S. and S. L. Lightman. 1992. Stress and the hypothalamo-pituitary-adrenal axis: Acute, chronic and immunological activation. J. Endocrinol. 134:327-339.

Hartsock, T. G. and R. A. Barczewski. 1997. Prepartum behavior in swine: effects of pen size. J. Anim. Sci. 75:2899-2904.

Jensen, P. 1994. Fighting between unacquainted pigs-Effects of age and of individual reaction pattern. Appl. Anim. Behav. Sci. 41:37-52.

Klieber, M. A., C. Underhill, G. L. Hammond and Y. A. Muller. 2007. Corticosteroid-binding globulin, a structural basis for steroid transport and proteinase-triggered release. J. Biol. Chem. 282:29594-29603.

Klont, R. E., B. Hulsegge, A. H. Hoving-Bolink, M. A. Gerritzen, E. Kurt, H. A. Winkelman-Goedhart, I. C. de Jong and R. W. Kranen. 2001. Relationships between behavioral and meat quality characteristics of pigs raised under barren and enriched housing conditions. J. Anim. Sci. 79:2835-2843.

Macleod, G. 1994. The flavour of beef. In: Flavor of Meat and Meat Product (Ed. F. Shahidi). pp. 4-37. Blackie Academic and Professional, Glasgow.

Maltin, C. A., C. C. Warkup, K. R. Matthews, C. M. Grant, A. D. Porter and M. I. Delday. 1997. Pig muscle fiber characteristics as a source of variation in eating quality. Meat Sci. 47:237-248.

McGough, C., N. Peacock, C. Heckett, C. Baldin, A. Norman, G. Frost, P. Blake, D. Tait, V. Khoo, K. Harrington, K. Whelan and H. J. N. Andreyev. 2006. Taste preferences for oral nutrition supplements in patients before and after pelvic radiotherapy: a double-blind controlled study. Clin. Nutr. 25:906-912.

Matsuki, Y. 2008. OIE world guidelines for animal welfare. Sustain. Livest. Prod. Hum. Welf. 62:3-9.

O'Connell, N. E., V. E. Beattie and B. W. Moss. 2004. Influence of social status on the welfare of growing pigs housed in barren and enriched environments. Anim. Welf. 13:425-431.

Persson, E., M. Wülbers-Mindermann, C. Berg and B. Algers. 2008. Increasing daily feeding occasions in restricted feeding strategies does not improve performance or well being of fattening pigs. Acta Vet. Scand. 50:24.

Petersen, V. 1994. The development of feeding and investigatory behavior in free-ranging domestic pigs during their first 18 weeks of life. Appl. Anim. Behav. Sci. 45:215-224.

Sather, A. P., S. D. M. Jones, A. L. Schaefer, J. Colyn and W. M. Robertson. 1997. Feedlot performance, carcass composition 
and meat quality of free-range reared pigs. Can. J. Anim. Sci. Westphal, U. 1986. Steroid-Protein Interactions II, Springer-Verlag, 77:225-232. Berlin, Germany.

Sato, S. 2011. Animal welfare: veterinarians' new role. J. Jpn. Vet. Yonezawa, T., M. Koori, T. Kikusui and Y. Mori. 2009. Appeasing Med. Assoc. 64:88-92.

Toko, K. 1996. Taste sensor with global selectivity. Mater. Sci. Eng. C. 4:69-82. pheromone inhibits cortisol augmentation and agonistic behaviors during social stress in adult miniature pigs. Zool. Sci. 26:739-744.

Toko, K. 1998. A taste sensor. Meas. Sci. Technol. 9:1919-1936.

Van der Wal, P. G., B. Engel and H. G. M. Reimert. 1999. The effect of stress, applied immediately before stunning, on pork quality. Meat Sci. 53:101-106.

Yonezawa, T., M. Irimajiri and A. Takahashi. JP Pat. Pend. No. 2011-50243 